\title{
Advances in Infrared Microprobe Instrumentation: Integration of Image Analysis and Molecular Spectroscopy
}

\author{
J. A. Reffner, J. A. Seelenbinder, and C. H. Tobler \\ Smiths Detection - Danbury, 14 \\ Commerce Drive, Danbury, CT 06810
}

Initially the infrared microprobe was developed to fill the spectroscopist's need to analyze sub-microgram samples of organic or inorganic covalent bonded materials; today, infrared microprobe analysis has become much more. Combining microscopy with infrared spectroscopy is a unique paring of microanalysis methods for solid-state characterization of molecular materials. Materials science is centered on relating structure with physical and chemical properties. Microstructure and microanalysis are essential analytical information for materials characterization. Imaging, metrology, and recording are needed to document microstructure; spectral analysis is needed to document chemistry. Electron microbeam analysis dominates studies of the elemental distributions in materials, but it is not as useful for molecular materials such as biologicals, drugs, fibers, minerals, and polymers. Integration of image analysis with molecular spectroscopy is a significant advance in infrared microprobe instrumentation.

The IlluminatIR II is the latest FT-IR spectrometer that can directly attach to many different commercial light microscopes and, when using special reflecting objectives converts, these microscopes into infrared microprobes. The IlluminatIR II is now driven by new software, SynchronizIR. SynchronizIR is an infrared microprobe data collection and archiving program that is built on Soft Imaging Systems (Munster, Germany), AnalySIS platform. SynchronizIR combines all image analysis functions of the AnalySIS platform with infrared spectral collection and analysis capabilities of Smiths Detection's SpecID software. Infrared spectra and images are archived together in a data base. When the microscope stage is motorized, automated analysis is possible.

In addition to conventional particle metrology, morphological features are used to direct the infrared analysis of particles. For example, a preparation containing a dispersion of cells is first surveyed and sorted by a size metric (such as diameter/ perimeter ratio) such that only circular cells with specific diameters are "selected" for spectral analysis. This automation is very important when a large number of spectra, of similar objects, are needed for chemometric analysis. The results of a solid-state phase analysis of sulfathiazole, showing three polymorphic forms, are presented in Figure 1.

The IlluminatIR II combines PLM with infrared spectroscopy, and when fitted with a heating, cooling or environmental stage, provides a microanalysis system for solid-state characterization. This combination is valuable for polymorph and stability screening of drugs substances and products. SynchronizIR is tailored to manage the Linkam heating stages (Linkam Scientific Instruments, Ltd., Surrey, U.K.). This software program lets the analyst setup a thermal cycle and automatically record a sequence infrared spectrum at selected temperatures; a micrograph may also be taken and linked with each spectrum. 
Dehydration of Carbamazepine is an example of a solid-state chemical reaction. The loss of water is seen in the $\mathrm{OH}$ stretching region (3300-3400 $\left.\mathrm{cm}^{-1}\right)$ and differences in bonding seen in the "fingerprint" spectral region $\left(800-1900 \mathrm{~cm}^{-1}\right)$. This data is reported in Figure 2.

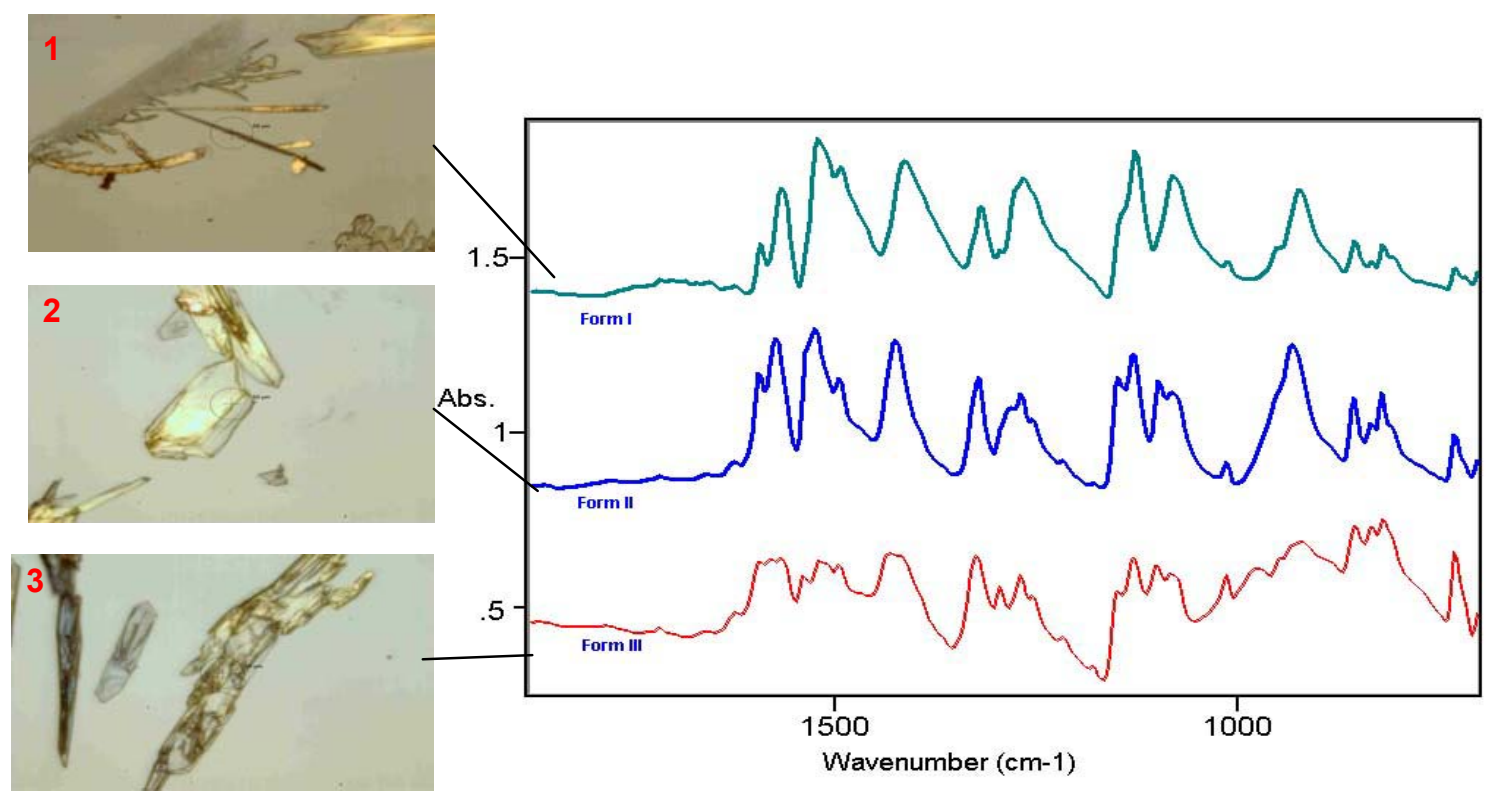

Figure 1. Sulfathiazole showing three polymorphic crystals forms. Crystals were automatically selected for infrared analysis using Image Directed Microanalysis.
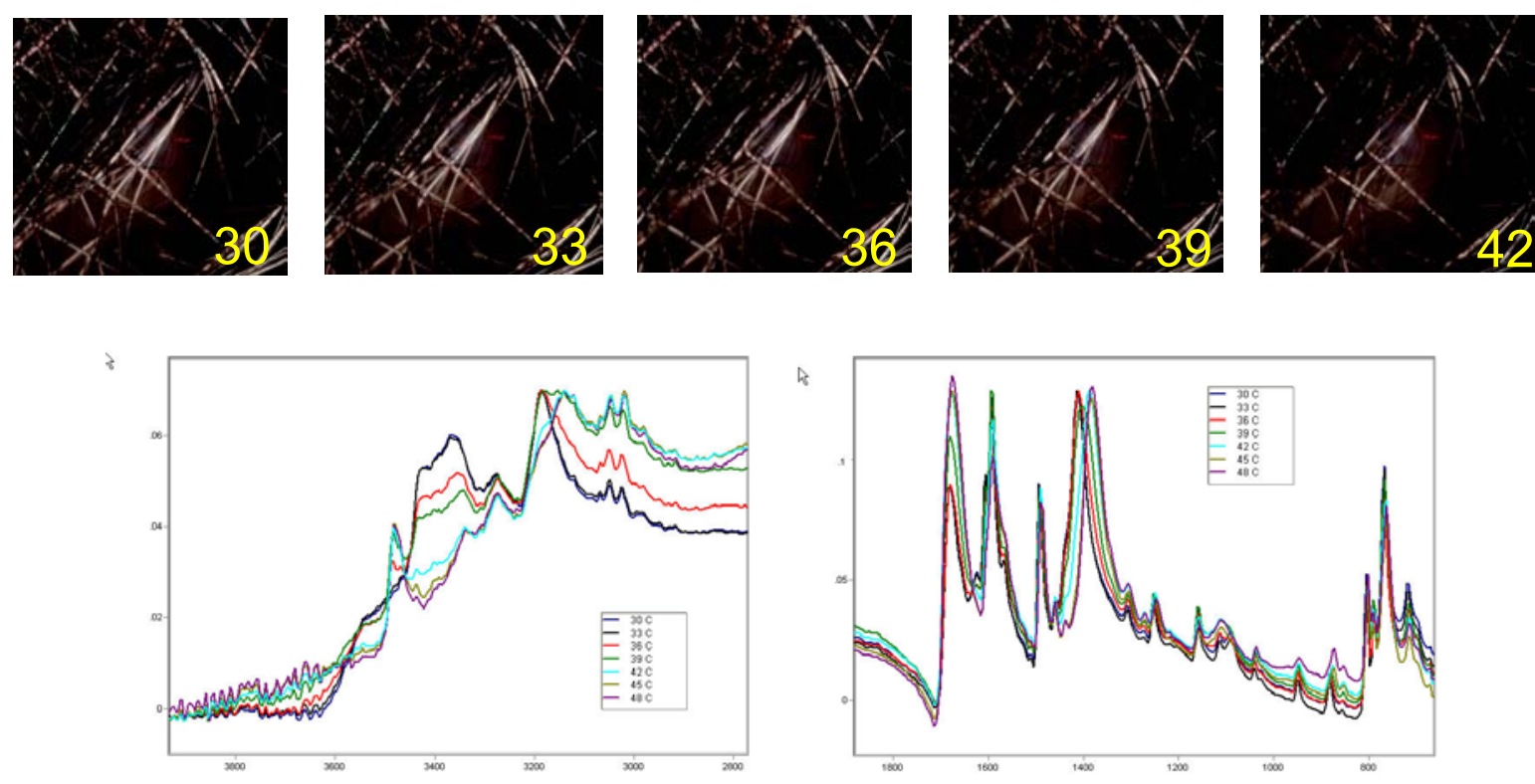

Figure 2. Dehydration of Carbamazepine 